\title{
Crosstalk in passive matrix polymer LED displays
}

\author{
D. Braun \\ Electrical Engineering Department. Cal Poly State University, San Luis Obispo, CA 93407. USA
}

\begin{abstract}
Passive matrix displays based on polymer and organic light-emitting diodes (LEDs) suffer from crosstalk, the emission of light from pixels other than those selected. This article explores the topic of crosstalk both with an approximate but analytic model and by means of more exact circuit simulation in order to explain the causes of crosstalk and to quantify the consequences. We conclude that crosstalk has implications for display power consumption.
\end{abstract}

Keywords: Electroluminescent displays; Polymer LEDs; Electrical crosstalk; Circuit simulation

\section{Introduction}

The discovery of electroluminescence in organic molecules [1] and semiconducting polymers [2] has generated considerable interest in display applications based on these materials. The materials make possible efficient, lightweight, thin, large area, flat-panel, and even mechanically flexible displays [3-5]. Although low-information-content indicators and displays are feasible, high-resolution passive matrix displays are among the most challenging uses envisioned, and several groups are working to overcome the technical hurdles [6-10].

Important technical issues must be resolved to create even a prototype computer monitor based on organic semiconductor materials. The topics span the gamut from chemical synthesis, solid-state physics, device physics, and fabrication processes to packaging and device degradation. All of these areas are the subject of active research [10]. One problem that has not received much attention is the issue of crosstalk, meaning that pixels other than the intended pixels emit light. The goal of this article is to explain this so-called crosstalk. We use circuit-modeling techniques to uncover and quantify several causes of crosstalk in passive matrix polymer lightemitting diode (LED) displays.

The allure of the technology is its simplicity. A polymer or organic LED pixel consists of multiple thin films deposited onto a glass substrate. Layers of organic materials are sandwiched between a metallic cathode and an inorganic anode, either indium tin oxide (ITO) or a thin metal. Applying a

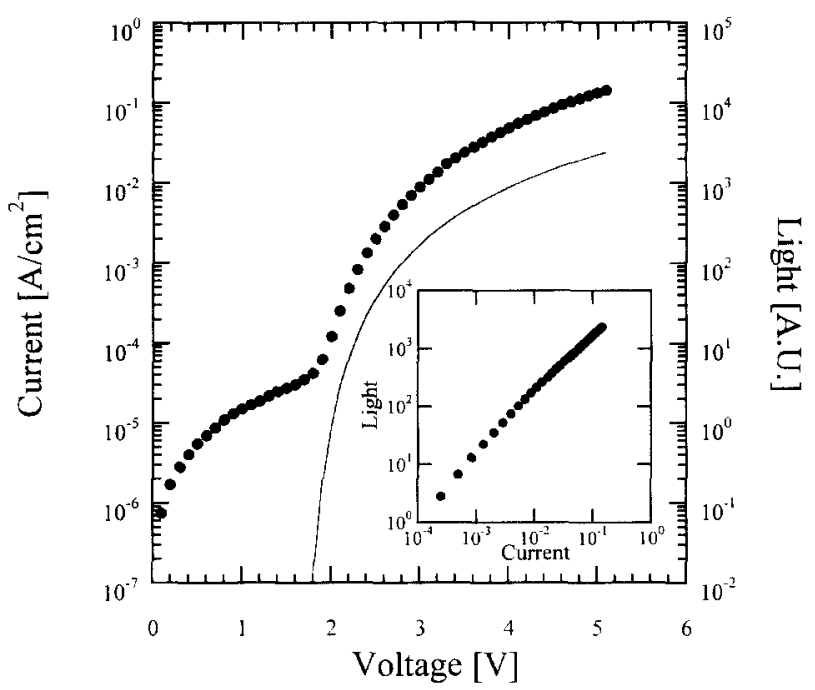

Fig. 1. Current (points) and light intensity (solid curve) as a function of voltage recorded for a polymer LED made from dialkoxy-PPV. The inset illustrates the linear dependence of light intensity on current.

positive voltage to the anode with respect to the cathode causes current flow in the pixel and light emission from the pixel. The device characteristics shown in Fig. 1 were obtained under nitrogen atmosphere from a device with an ITO anode, a dialkoxy-polyphenylene vinylene (PPV) emission layer, and a $\mathrm{Ca}$ cathode. The data are similar to those already available in the literature [11-13]. Emission efficiencies above $1 \%$ photons/electron are typical.

A passive matrix display consists of an array of pixels with pixels in the same row sharing a common cathode electrode and pixels in the same column sharing a common anode 


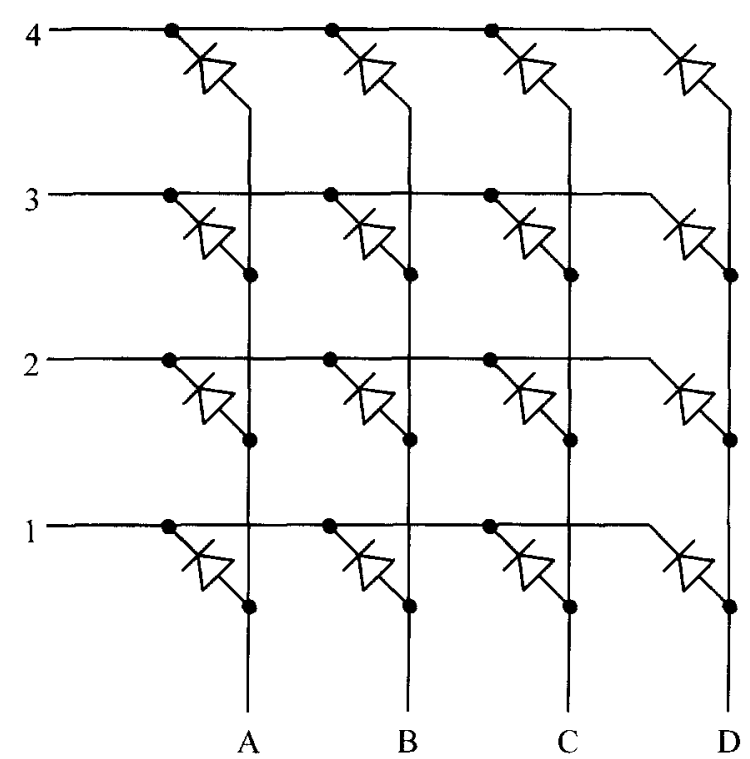

Fig. 2. Passive matrix array of LEDs. Rows 1 through 4 form the cathode electrodes, and columns $\mathrm{A}$ through $\mathrm{D}$ form the anode electrodes.

electrode. Fig. 2 illustrates the electrical connections for a $4 \times 4$ pixel display. In theory, applying a positive voltage to a column electrode with respect to a row electrode will turn on the LED located at the intersection between the two electrodes and cause light emission from one pixel. In practice, other pixels emit light as well. Consider LED Al at the intersection of column A and row 1. Applying a positive bias to column A with respect to row 1 should energize LED A 1 only. Experiments show that other pixels in column A (A2, A3, or A4) and row 1 (B1, C1, or D1) can produce unwanted light.

Fig. 3 hints at one likely cause of the crosstalk. Fig. 3 contains the same matrix of LEDs as Fig. 2, but the pixels are presented in a circuit topology that emphasizes the conduction paths parallel to the one selected pixel A1. The conduction paths consist of three pixels in series with two LEDs oriented with the same polarity and one oriented with the opposite polarity. A forward-bias voltage across pixel Al would appear across series combinations such as A2, B2, and $\mathrm{B} 1 ; \mathrm{A} 2, \mathrm{C} 2$, and $\mathrm{C} 1$; and $\mathrm{A} 4$; $\mathrm{B} 4$, and $\mathrm{B} 1$. In each case, two

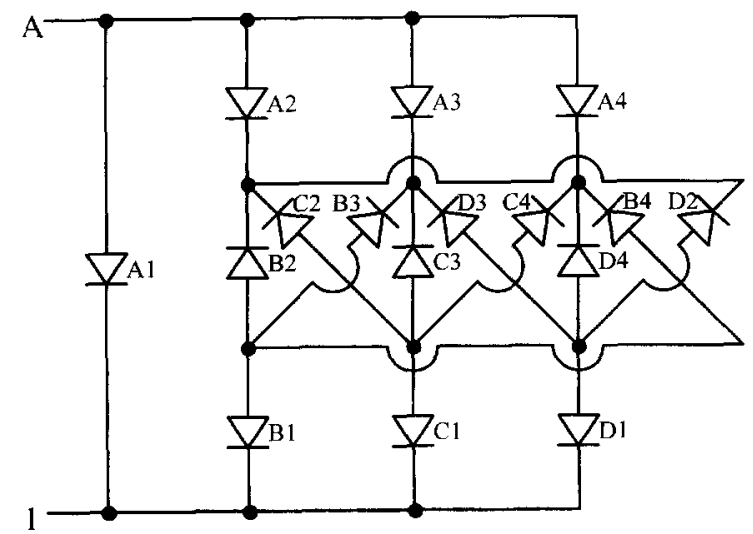

Fig. 3. Passive matrix array of LEDs draws so as to emphasize conduction paths parallel to one selected LED.
LEDs are forward biased and one is reverse biased. With LEDs acting like ideal diodes, the reverse-biased LEDs would not permit any current flow, and no light could emerge from the forward-biased LEDs. Real LEDs do permit some current to flow under reverse bias, so several reverse-biased LEDs in parallel can pass enough current to allow light emission from another LED in series. For example, pixel A2 lies in series with pixels B2, C2, and D2. Even a low-information-content display would have more than $4 \times 4$ pixels, so more parallel conduction paths exist.

The purpose of this work is to quantify processes that contribute to crosstalk using circuit simulation of a passive matrix based on the electrical properties of a single pixel. Although optical crosstalk due to the internal reflection of the light is also of concern [14], we consider only electrical crosstalk. Additionally, we assume that connections between pixels are formed from zero-resistance wires. Before obtaining quantitative results from circuit simulation, however, it is useful to anticipate results with a heuristic approach.

\section{Sources of crosstalk}

There are several potential sources of d.c. crosstalk in LED matrix displays. After defining these sources, we compare their influence on crosstalk by determining the intensity of stray light created by other pixels relative to the intensity emitted by the selected pixel.

- Display resolution: the greater number of pixels required for higher-resolution displays creates more available parallel conduction paths, so one would predict more stray light in higher-resolution displays.

- Rectification ratio: one rough way to characterize diode quality is in terms of the ratio of current flowing in forward bias to that flowing under the same magnitude reverse bias. A higher rectification ratio indicates that less leakage current flows for a given magnitude bias, so less stray light should occur with higher-rectification devices.

- Reverse leakage current: another way to characterize the reverse-bias quality of a diode is in terms of the parallel leakage paths that compromise the diode action under reverse bias. Larger reverse leakage current would increase the stray light generated.

\section{Analytic modeling results}

Back-of-the-envelope calculations can provide an approximate understanding of the influences of display resolution and rectification ratio on crosstalk. For example, based on an analytic expression for the electrical characteristics of each pixel, circuit analysis can generate a means of determining the stray light. Let us begin by finding the stray light emitted as a function of positive voltage $V_{\mathrm{A} 1}$ applied across pixel A1 in Fig. 2 (or Fig. 3). The symmetry of Fig. 3 shows that any of the other forward-biased pixels in column $\mathrm{A}$ and row 1 are 
candidates to produce stray light, and they are equivalent candidates. It is safe to assume that a reverse-biased pixel does not emit light, so we only really need to calculate the stray light emitted by pixel A2.

The data of Fig. 1 can supply an approximate expression that describes the electrical characteristics of each pixel. The current versus voltage curve of Fig. 1 consists of two curved sections. Below a threshold voltage of approximately $1.8 \mathrm{~V}$, only a leakage current flows. Above the threshold voltage, the current increases as a power law with respect to the voltage, and the light emission is proportional to the current. If this pixel sits at the intersection of column $C$ and row $R$ of the matrix, the approximate expression for current flow through the pixel is

$I_{C R}=I_{0} V_{C R}^{b}$

where $I_{C R}$ is the current flow through the pixel and $V_{C R}$ is the voltage drop across the pixel. A more accurate treatment would account for the threshold voltage, but we will use circuit simulation in Section 4 to obtain more exact quantitative results based on the experimental data. In order to assess the influence of rectification ratio on the crosstalk, the roughest and least conservative approximation would be to assume a constant rectification ratio, $r$, as a function of voltage. Then the current flow under reverse bias would have the expression

$I_{C R}=-\frac{I_{0}\left|V_{C R}\right|^{b}}{r}$, when $V_{C R}<0$

(A) Consider the case with pixel Al forward biased $\left(V_{\mathrm{A} 1}>0\right)$. Then

$V_{\mathrm{A} 1}=V_{\mathrm{A} 2}-V_{\mathrm{B} 2}+V_{\mathrm{B} 1}=2 V_{\mathrm{A} 2}-V_{\mathrm{B} 2}$

For an array with $N$ rows and $N$ columns $(N>2)$,

$V_{\mathrm{B} 2}=-\left[V_{\mathrm{A} 1}-2 V_{\mathrm{A} 2}\right]=-V_{\mathrm{A} 1}+2\left[(N-1) I_{\mathrm{B} 2} / I_{0}\right]^{1 / b}$

$$
=-V_{\mathrm{A} 1}-2\left(\frac{N-1}{r}\right)^{1 / b} V_{\mathrm{B} 2}
$$

Solve for the voltage drop across pixel B2 to obtain

$V_{\mathrm{B} 2}=\frac{-V_{\mathrm{A} 1}}{1+2\left(\frac{N-1}{r}\right)^{1 / b}}$

Combine Eqs. (3), (4), and (5) to obtain the voltage drop across pixel A2,

$V_{\mathrm{A} 2}=\frac{V_{\mathrm{A} 1}}{2+\left(\frac{r}{N-1}\right)^{1 / b}}$

The current flow through pixel $\mathrm{A} 2$ is

$$
I_{\mathrm{A} 2}=I_{0} V_{\mathrm{A} 2}^{b}=\frac{I_{0} V_{\mathrm{A} 1}^{b}}{\left[2+\left(\frac{r}{N-1}\right)^{1 / b}\right]^{b}}
$$

The ratio of the stray light emitted by pixel A2 to the light emitted by pixel $\mathrm{Al}$ is proportional to the ratio of the currents:

$\frac{L_{\mathrm{A} 2}}{L_{\mathrm{A} 1}}=\frac{I_{\mathrm{A} 2}}{I_{\mathrm{A} 1}}=\frac{1}{\left[2+\left(\frac{r}{N-1}\right)^{1 / b}\right]^{b}}$

The total stray light, $L_{\mathrm{T}}$, emitted by the display is obtained from the sum of the light emitted by pixels in column $\mathrm{A}$ and row 1 other than pixel A1:

$\frac{L_{\mathrm{T}}}{L_{\mathrm{A} 1}}=\frac{2(N-1) I_{\mathrm{A} 2}}{l_{\mathrm{A} 1}}=\frac{2(N-1)}{\left[2+\left(\frac{r}{N-1}\right)^{1 / b}\right]^{b}}$

Eqs. (8) and (9) indicate two trends. A higher rectification ratio, $r$, increases the denominators and decreases the amount of stray light emitted. For large $r$, for example, the stray light ratio is proportional to $1 / r$, so it pays to use highly rectifying devices. The more rows and columns, $N$, the more stray light is emitted, because more parallel paths exist to supply current to pixels in column A and row 1 (other than pixel A1). Figs. 4 and 5 plot these analytical results along with those obtained from simulation, so the discussion is deferred until Section 4 .

(B) Consider the case with pixel Al reverse biased $\left(V_{\mathrm{A} I}<0\right)$, so pixels in row 1 or column $\mathrm{A}$ are reverse biased. Stray light could leak from the pixels in other rows and columns, which would be forward biased. Eq. (4) no longer applies, rather

$$
\begin{aligned}
V_{\mathrm{B} 2} & =-\left[V_{\mathrm{A} 1}-2 V_{\mathrm{A} 2}\right]=-V_{\mathrm{A} 1}-2\left[r(N-1) I_{\mathrm{B} 2} / I_{0}\right]^{1 / b} \\
& =-V_{\mathrm{A} 1}-2[r(N-1)]^{1 / b} V_{\mathrm{B} 2} \\
& =\frac{-V_{\mathrm{A} 1}}{1+2[r(N-1)]^{1 / b}}
\end{aligned}
$$

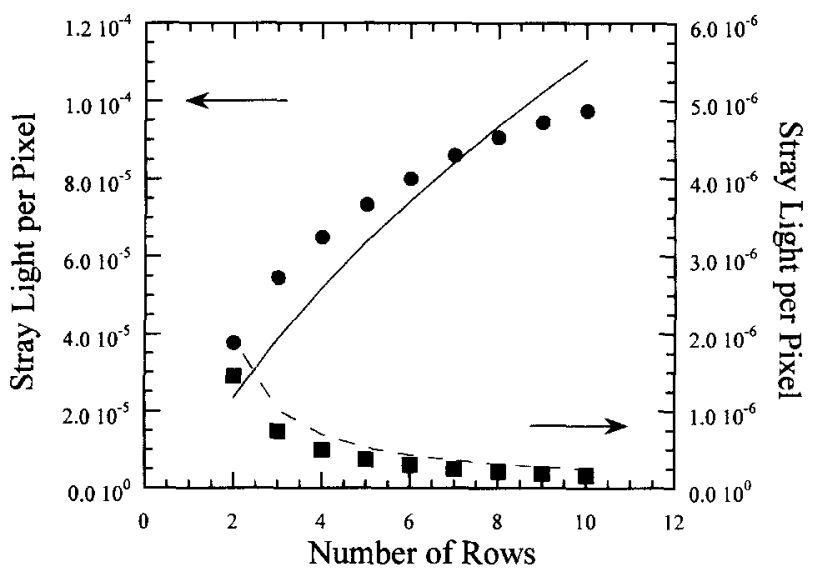

Fig. 4. Stray light per pixel as a function of display size with rectification ratio $r=10^{4}$. Stray light is quantified as the unwanted light intensity emitted by one pixel normalized to the light intensity emitted by an addressed pixel biased to $+5 \mathrm{~V}$. Round data points are simulation results for pixel Al forward biased with light emitted from pixel A2, and the solid curve is the least-squares fit to Eq. (8) with $b=5.11$. Square data points are simulation results for pixel A1 reverse biased with light emitted from pixel $\mathrm{B} 2$, and the dashed curve is Eq. (11) with $b=5.11$. 


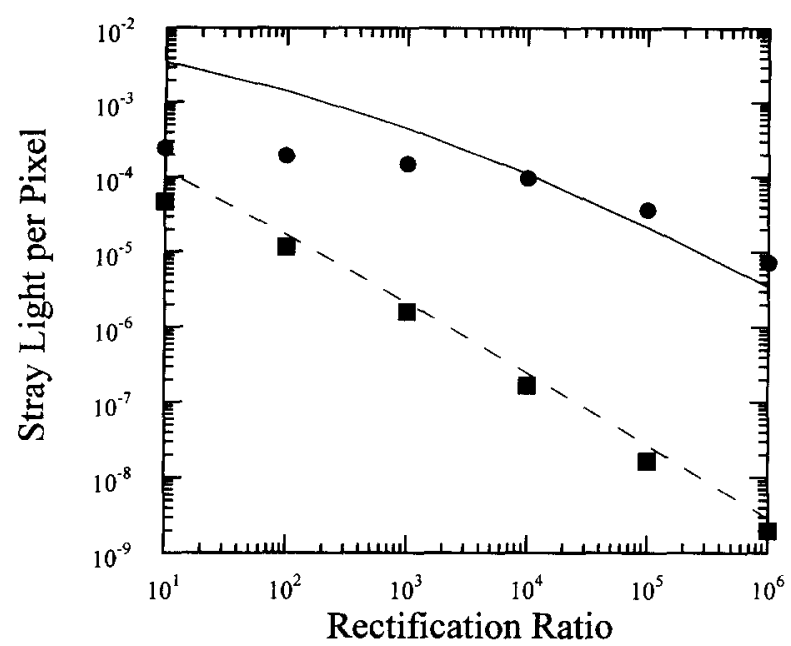

Fig. 5. Stray light per pixel as a function of rectification ratio for a display with 10 rows and 10 columns. Round data points are simulation results for pixel Al forward biased with light emitted from pixel A2, and the solid curve is $\mathrm{Eq}$. (8) with $b=5.11$. Square data points are simulation results for pixel A1 reverse biased with light emitted from pixel B2, and the dashed curve is Eq. (11) with $b=5.11$.

Then, since the reverse-biased pixel A1 would not emit light, it is more useful to compare the stray light emitted from pixel B2 to the light that would be emitted from pixel A1 under the same magnitude forward bias:

$\frac{L_{\mathrm{B} 2}}{L_{\mathrm{A} 1}}=\frac{I_{\mathrm{B} 2}}{I_{\mathrm{A} 1}}=\frac{1}{\left\{1+2[r(N-1)]^{1 / b}\right\}^{b}}$

The total stray light emitted by all of the forward-biased pixels in the display, that is, all of the pixels outside of column A and row 1, becomes

$\frac{L_{\mathrm{T}}}{L_{\mathrm{A} 1}}=\frac{(N-1)^{2} I_{\mathrm{B} 2}}{I_{\mathrm{A} 1}}=\frac{(N-1)^{2}}{\left\{1+2[r(N-1)]^{1 / b}\right\}^{b}}$

The trends that emerge from Eqs. (11) and (12) differ slightly from the trends under forward bias. As in forward bias, the stray light decreases for larger rectification ratios. Unlike in forward bias, the stray light per pixel also decreases as the number of columns and rows increases, because more rows and columns create more parallel paths that connect column A to row 1. The result is less current (and light) per path.

(C) Pixels with leakage current under reverse bias.

The simple model used above does not account for any reverse-bias leakage current other than that allowed by the rectification of the diode. If, for example, each reverse-biased pixel behaves as a resistor with resistance $R$, then the ratio of stray light will also depend on the applied voltage.

The derivation proceeds in the same manner as that leading up to Eqs. (8) and (11), and the pixel model assumes an infinite rectification ratio. For pixel $\mathrm{A} 1$ forward biased, it can be shown that the ratio of stray light generated in other forward-biased pixels in column A or row 1 is
$\frac{L_{\mathrm{A} 2}}{L_{\mathrm{A} 1}}=\frac{I_{\mathrm{A} 2}}{I_{\mathrm{A} 1}}=\frac{V_{\mathrm{A} 2}^{b}}{V_{\mathrm{A} 1}^{b}}=\frac{1}{\left[2+\frac{R I_{0}}{N-1} V_{\mathrm{A} 2}^{b-1}\right]^{b}}$

For pixel A1 reverse biased, it can be shown that the ratio of stray light generated in other forward-biased pixels not in column A or row 1 is

$\frac{L_{\mathrm{B} 2}}{L_{\mathrm{A} 1}}=\frac{I_{\mathrm{B} 2}}{I_{\mathrm{A} 1}}=\frac{V_{\mathrm{B} 2}^{b}}{V_{\mathrm{A} 1}^{b}}=\frac{1}{\left[1+2 R(N-1) I_{0} V_{\mathrm{B} 2}^{b-1}\right]^{b}}$

As less current flows under reverse bias (greater $R$ ), less stray light results. Also, the influence of display resolution follows the same trend as before. The stray light ratio increases with the voltage drop across the relevant pixels, but the ratio of stray light to intended light decreases at higher voltage levels.

\section{Circuit simulation results}

While the equations derived in Section 3 can make qualitative predictions, circuit simulation can obtain more exact results by working from a more accurate version of the electrical data rather than a numerical approximation. The approach used is to insert the electrical data for $1 \mathrm{~cm}^{2}$ LEDs from Fig. 1 directly into the circuit simulation tool Pstar ${ }^{\mathrm{TM}}$ and enter a circuit net list consisting of an $N \times N$ array of pixels like that shown in Fig. 2.

Fig. 4 shows the stray light ratio per pixel as a function of array size. The voltages applied to pixel $\mathrm{Al}$ are $\pm 5 \mathrm{~V}$. The pixels have the forward-bias characteristics shown in Fig. 1 and a rectification ratio of $10^{4}$. As in Eq. (8) for the forwardbias case, the stray light ratio increases in higher-resolution arrays. The reverse-bias case reflects the trend of Eq. (11) with the stray light per pixel decreasing with array size. Note that the reverse-bias stray light is about two orders of magnitude smaller than the forward-bias stray light, mainly for two reasons:

(1) Each conduction path in parallel with the selected pixel A1 contains two diodes biased with the same polarity and one biased with opposite polarity. Forward-bias stray light arises when only one of the three pixels is reversed biased, and reverse-bias stray light arises when two of the three pixels are reverse biased. For the same magnitude bias on pixel A 1 , more reverse current can flow through the stray light path when only one pixel is blocking current.

(2) In forward bias, each forward-biased pixel that could emit light receives its current from $(N-1)$ reverse-biased pixels, whereas under reverse bias, each forward-biased pixel is sandwiched between two reverse-bias pixels and is one of $(N-1)$ pixels vying for the meager current available.

Fig. 5 illustrates the effect of rectification on stray light generation. In forward bias, there is an exponential incentive to increase the rectification of each pixel. Reverse bias allows less stray light with low rectification ratios, and reasonable 
rectification ratios suppress the stray light by additional orders of magnitude.

Note that the calculations in part (A) treat each pixel in the array identically, which is a worthwhile goal in production. It is more realistic to consider that faults occur in occasional pixels. The goal is to determine the symptoms caused by certain faults such as short circuits with an eye to designing tests that could diagnose the faults. We consider the effect of one short-circuited pixel in Figs. 6 and 7 and the effects of two shorts in Fig. 8. All short-circuit simulation results use a $10 \times 10$ pixel array of LEDs having the electrical characteristics shown in Fig. 1 and rectification ratios of $10^{4}$. Note that simulation data at high light intensities ( $>2500$ A.U.) are

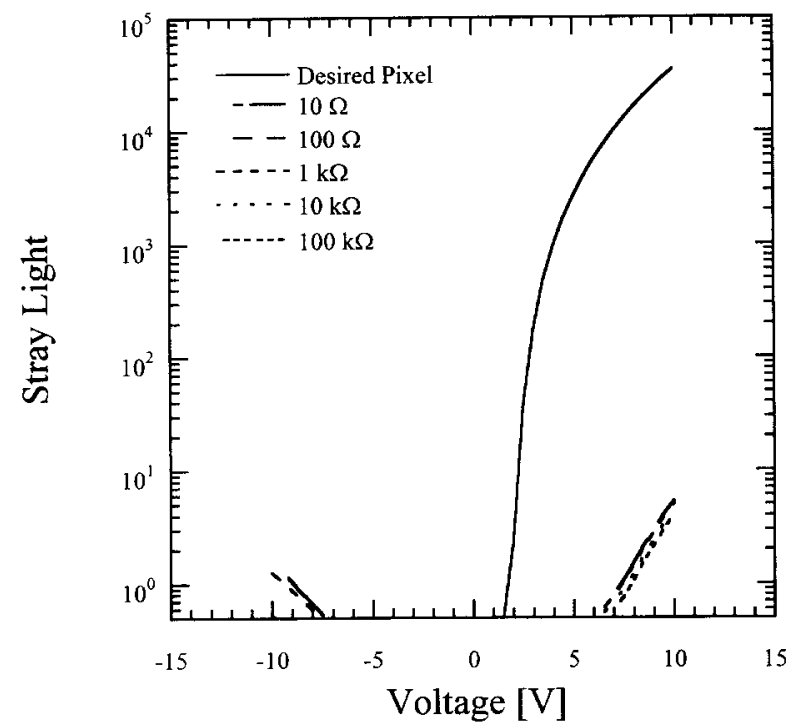

Fig. 6. Stray light per pixel as a function of the voltage applied to the selected pixel (A1) for several values of short-circuit resistance in the selected row (e.g., pixel BI). The solid curve is light intensity as a function of bias for the selected pixel.

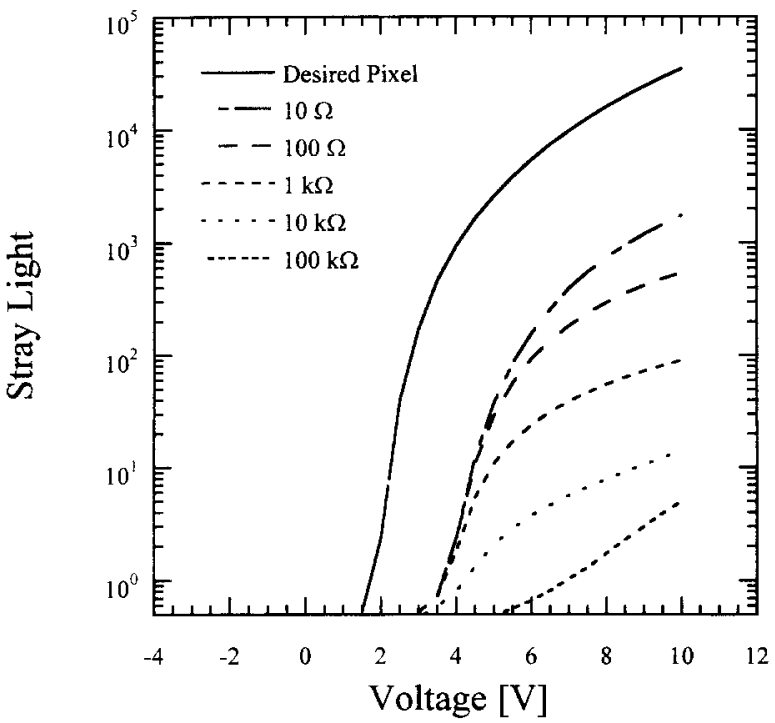

Fig. 7. Stray light per pixel as a function of the voltage applied to the selected pixel (A1) for several values of short-circuit resistance in a blocking pixel (e.g., pixel B2). The solid curve is light intensity as a function of bias for the selected pixel.

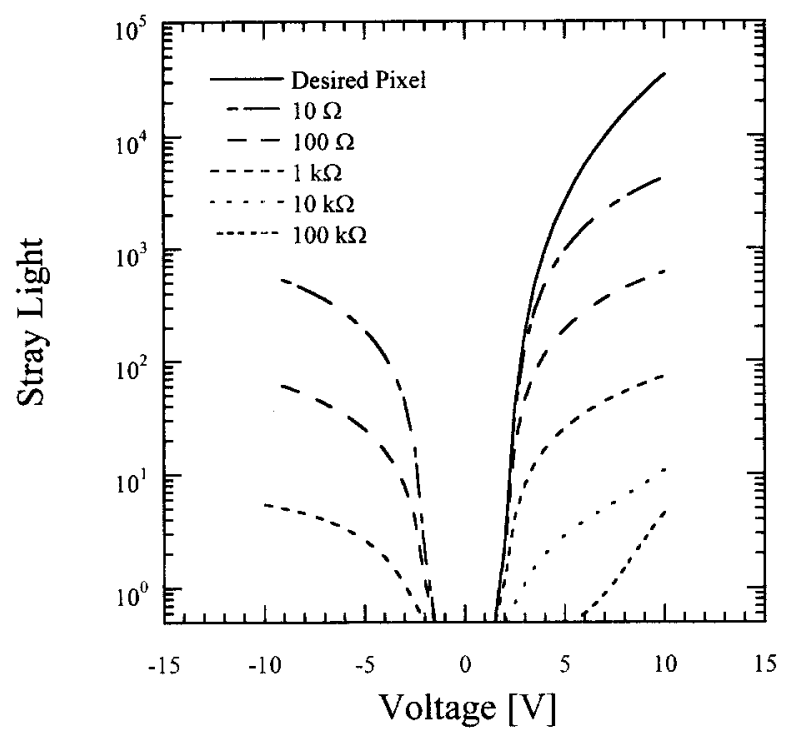

Fig. 8. Stray light per pixel as a function of the voltage applied to the selected pixel (A1) for several values of short-circuit resistance in two pixels. The solid curve is light intensity as a function of bias for the selected pixel.

extrapolated from data expected for pulsed LED operation [15] rather than from the d.c. characteristics of Fig. 1.

Fig. 6 shows that one short circuit in the selected row causes little stray light generation, because at least one pixel in each undesired conduction path remains reverse biased to block current. The data are calculated with one pixel in the selected row (B1) replaced by a resistor. Each other pixel has a rectification ratio of $10^{4}$. With a forward bias applied to the selected pixel A1, pixels such as A2 in the same column as the selected pixel may generate light. With a reverse bias applied to the selected pixel Al, the stray light comes from pixels such as B2 in other rows and columns. The effect is minimal when the short circuit occurs in a pixel that would be forward biased.

Fig. 7 shows that a short circuit in a non-selected row can allow stray light by disabling the blocking action of the reverse-biased pixel. The data are calculated with one pixel in a non-selected row (B2) replaced by a resistor. With a forward bias applied to the selected pixel A1, the stray light comes from pixels in the same column (A2). With a reverse bias applied to the selected pixel $\mathrm{A} 1$, the stray light comes from pixels in other rows and columns (B2).

Fig. 8 shows the dramatic effect of short circuits occurring simultaneously in two rows. For the selected pixel (Al) forward biased, one short circuit in the selected row (B1) combined with one short circuit in another row (B2) allows the then forward-biased pixel in the selected column (A2) to emit a significant amount of stray light. The disastrous result for the selected pixel (A1) reverse biased occurs when shorts occur both in the selected row (B1) and in the selected column (A2). The one pixel that connects the two shorts (B2) emits significant amounts of light, because it finds itself forward biased. Stray light emitted with the desired pixel reverse biased will likely be visible, because the desired pixel emits no light under reverse bias. These data explain unusual 
situations observed in the laboratory. For example, with a negative voltage applied to pixel A I, pixel D4 shines brightly.

\section{Discussion}

While the absolute magnitude of stray light generated per pixel in most of the cases considered does not appear alarming, the eye is a sensitive detector. At sufficient brightness the data in Figs. 7 and 8 show that unintentionally addressed pixels can emit stray light at easily visible intensities above $1 \%$ of the desired pixel's intensity. Also, the total stray light emitted by the array is another factor of $(N-1)$ to $(N-1)^{2}$ greater. The stray light can represent a significant power drain on a display.

Following the reasoning of Section 3 provides rough estimates of the power dissipation. Beginning with Eq. (8), we obtain the total power dissipation $P_{\mathrm{T}}$ as a function of rectification ratio and display resolution for the case with the selected pixel Al forward biased:

Table 1

Power dissipation in watts as a function of rectification ratio

\begin{tabular}{lll}
\hline Rectification ratio & Selected pixel $@+10 \mathrm{~V}$ & Selected pixel @ $-10 \mathrm{~V}$ \\
\hline $10^{1}$ & 19.39 & $1.39 \times 10^{0}$ \\
$10^{2}$ & 18.35 & $2.25 \times 10^{-1}$ \\
$10^{3}$ & 17.93 & $2.63 \times 10^{-2}$ \\
$10^{4}$ & 17.82 & $2.89 \times 10^{-3}$ \\
$10^{5}$ & 17.80 & $2.96 \times 10^{-4}$ \\
$10^{6}$ & 17.80 & $2.97 \times 10^{-5}$ \\
\hline
\end{tabular}

$$
\begin{aligned}
P_{\mathrm{T}} & =V_{\mathrm{A} 1} I_{\mathrm{A} 1}+(N-1) V_{\mathrm{A} 1} I_{\mathrm{A} 2}=I_{0} V_{\mathrm{A} 1}^{b+1}+(N-1) V_{\mathrm{A} 1} I_{0} V_{\mathrm{A} 2}^{b} \\
& =I_{0} V_{\mathrm{A} 1}^{b+1}+\frac{(N-1) I_{0} V_{\mathrm{A} 1}^{b+1}}{\left[2+\left(\frac{r}{N-1}\right)^{1 / b}\right]^{b}}
\end{aligned}
$$

Similarly, with the selected pixel A1 reverse biased:

$$
\begin{aligned}
P_{\mathrm{T}} & =V_{\mathrm{A} 1} I_{\mathrm{A} 1}+(N-1)^{2} V_{\mathrm{A} 1} I_{\mathrm{B} 2} \\
& =\frac{I_{0} V_{\mathrm{A} 1}^{b+1}}{r}+\frac{(N-1)^{2} I_{0} V_{\mathrm{AI}}^{b+1}}{\left\{1+2[r(N-1)]^{1 / b}\right\}^{b}}
\end{aligned}
$$

The data in Table 1, obtained from the simulation results of Section 4 on a $10 \times 10$ pixel array with a total pixel area of $100 \mathrm{~cm}^{2}$, bear out the predictions of Eqs. (15) and (16). With the selected pixel forward biased, the rectification ratio does moderately change the power dissipation. With the selected pixel reverse biased, the rectification ratio effects quiescent power dissipation with the power dissipation almost proportional to the reciprocal of the rectification ratio.

We use the simulation results with rectification ratio $r=10^{4}$ to determine the power dissipation as a function of shortcircuit placement and resistance. Tables 2 and 3 contain the power dissipation for the selected pixel forward biased and reverse biased for the cases considered in Figs. 6-8. The power dissipation of the display with the selected pixel forward biased does not depend significantly on the short-circuit resistance, except for the most extreme case of two low-resistance shorts in series. Table 3 shows that the reversebias selection depends more strongly on short-circuit resistance and placement. With two blocking diodes in the majority of the parallel conduction paths, the total power

Table 2

Power dissipation in watts for selected pixel A 1 forward biased to $+10 \mathrm{~V}$

\begin{tabular}{lllll}
\hline Short-circuit resistance & Short in selected row & Short in blocking pixel & Shorts in selected row + another & Shorts in selected row + column \\
\hline $10 \Omega$ & 17.82 & 18.71 & 20.00 & 17.83 \\
$100 \Omega$ & 17.82 & 18.10 & 18.13 & 17.83 \\
$1 \mathrm{k} \Omega$ & 17.82 & 17.86 & 17.85 & 17.83 \\
$10 \mathrm{k} \Omega$ & 17.82 & 17.83 & 17.82 & 17.82 \\
$100 \mathrm{k} \Omega$ & 17.82 & 17.82 & 17.82 & 17.82 \\
$x$ & 17.82 & 17.82 & 8 & 17.82 \\
See Figure & 6 & 7 & B1 \& B2 & 8 \\
Pixel & B1 & B2 & & B1 \& A2 \\
\hline
\end{tabular}

Table 3

Power dissipation in milliwatts for selected pixel Al reverse biased to $-10 \mathrm{~V}$

\begin{tabular}{llllc}
\hline Short-circuit resistance & Short in selected row & Short in blocking pixel & Shorts in selected row + another & Shorts in selected row + column \\
\hline $10 \Omega$ & 9.36 & 2.90 & 10.3 & 2190 \\
$100 \Omega$ & 9.14 & 2.90 & 10.0 & 320 \\
$1 \mathrm{k} \Omega$ & 7.66 & 2.90 & 8.23 & 39.8 \\
$10 \mathrm{k} \Omega$ & 4.50 & 2.90 & 4.63 & 6.86 \\
$100 \mathrm{k} \Omega$ & 3.08 & 2.89 & 3.08 & 3.25 \\
$\infty$ & 2.89 & 7.89 & 8 & 8.89 \\
See Figure & 6 & B2 & B1 \& B2 & B1 \& A2 \\
Pixel & B1 & & & \\
\hline
\end{tabular}


dissipation is much lower than in the situation under forward bias in which at most one blocking diode sits in series to reduce leakage current.

The desire to facilitate testing provides another motivation to model floating electrode matrices. Because of the large number of electrical connections involved for even a moderate resolution display, one would like to find an experimental technique to characterize $N \times M$ pixel displays without having to form $N+M$ electrical connections. In a square matrix, it might be possible to test the display for faults with fewer than $N$ measurements. After the testing process determines that each pixel functions properly, image uniformity becomes the primary concern. Because of the crosstalk issues outlined above for a display having floating electrodes, controlling the image on a display usually entails controlling the potential at all electrodes. While beyond the scope of this article, a similar analysis of image uniformity is in progress for images formed using a line-scan technique in which drivers select one row of pixels at a time.

\section{Conclusions}

Much interest in polymer and organic light-emitting diodes (LEDs) derives from their potential to deliver the next generation of flat passive matrix displays. The devices are relatively easy to make, because they consist of an electroluminescent layer sandwiched between an anode, usually transparent, and a cathode. A passive matrix display results by patterning the anode into columns and the cathode into rows to form an array of pixels from the intersections between the cathode and anode electrodes. Applying a positive bias to one anode and a negative bias to one cathode causes light emission from the pixel shared by the two electrodes. In many cases, light also emerges from other pixels in the matrix. The crosstalk has several origins. Viewing the large numbers of pixels in a matrix as potential parallel conduction paths hints at how crosstalk can arise. This work has analyzed how crosstalk depends on display resolution, the degree of rectification of the individual LEDs, and, most significantly, on the reverse leakage current of the pixels. One faulty pixel can cause crosstalk, and crosstalk can be quite severe with as few as two faulty pixels. The reverse-bias characteristics of the LED hold one key to suppressing crosstalk, so low current flow in reverse bias is essential.

\section{Acknowledgements}

The modeling work was performed at Philips Research Laboratories in Eindhoven, The Netherlands, where Dr Coen Liedenbaum provided the device data shown in Fig. 1. The Cal Poly College of Engineering Investment in Faculty Program supported the preparation of this manuscript.

\section{References}

[1] C.W. Tang and S.A. VanSlyke, Appl. Phys. Lett., 51 (1987) 913-915.

[2] J.H. Burroughes, D.D.C. Bradley, A.R. Brown, R.N. Marks, K. Mackay, R.H. Friend, P.L. Burns and A.B. Holmes, Nature, 347 (1990) 539-541.

[3] D. Braun and A.J. Heeger, Appl, Phys. Lett., 58 ( 1991) 1982-1984.

[4] G. Gustafsson, Y. Cao, G.M. Treacy, F. Klavetter, N. Colaneri and A.J. Heeger, Nature, 357 (1992) 477-479.

[5] Y. Yang, MRS Bull., 22 (1997) 31-38.

[6] P. May, Society for Information Display 96 Digest, San Diego, CA, 1996, pp. 192-195.

[7] R.F. Service, Science, 273 ( 1996) 878-880.

[8] J.R. Sheats, H. Antoniadis, M. Hueschen, W. Leonard, J. Miller, R. Moon, D. Roitman and A. Stocking, Science, 273 ( 1996) 884-888.

[9] A. Shen, P.E. Burrows and M.E. Thompson, Science, 276 (1997) 2009.

[10] Proc. 1996 Int. Conf. Sci. Technol. Synthetic Metals, Synth. Met., 85 (1997) 1173-1444

[11] D. Braun, E.G.J. Staring, R.C.J.E. Demandt, G.L.J. Rikken, Y.A.R.R Kessener, A.H.J. Venhuizen and M. Bouwmans, Synth. Met., 66 (1994) 75-79.

[12] G. Yu, K. Pakbaz and A.J. Heeger, J. Electron. Mater., 23 ( 1994) 925

[13] P.W.M. Blom, M.J.M. de Jong, C.T.H.F. Liedenbaum and J.J.M Vleggaar, Synth. Met., 85 (1997) 1287-1288.

[14] W.N. Jacobus and S.-M. Ku, US Patent No. 3846193 (1974).

[15] D. Braun, D. Moses, C. Zhang and A.J. Heeger, Appl. Phys. Lett., 61 (1992) 3092-3094. 